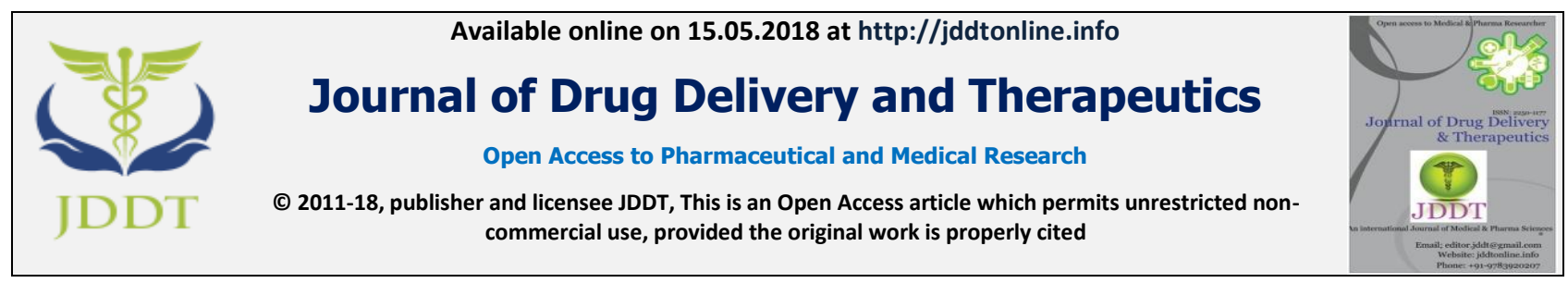

Open $\odot$ Access

Research Article

\title{
IDENTIFICATION OF BIOLOGICAL COMPONENTS FROM POTENTIAL BONE HEALER MEDICINAL PLANTS
}

\author{
Sabeerali Ansarali, Selvarasuvasuki Manikandan and Ganapathy Murugan Alagu Lakshmanan* \\ Department of Botany, Annamalai University, Annamalainagar - 608 002, Tamil Nadu, India \\ *Department of Botany, Thiru Kolanjiappar Govt. Arts College, Vriddhachalam, India - 606001.
}

\section{ABSTRACT}

Nature has been a wellspring of therapeutic for a vast number of years and a great number of present day drugs have been separated from plant resources. It has been evaluated that natural prescriptions serve around $80 \%$ of the total population wellbeing requirement for many individuals throughout the world. Cissus quadrangularis a member of the family Vitaceae, Cardiospermum halicacabum and Dodonea viscosa members of the family Sapindaceae are well known for its utilization in the treatment of skin contaminations, blockage, piles, lack of health, weight loss, ant-inflammatory, anti-ulcer and bone crack healing. In the present study, based on GCMS methanolic extract of stem and leaf of these three plants showed and identified fifteen major compounds with pharmacological activities. The FT-IR analysis showed similar functional groups of compounds related their medicinal properties such as Bone healing and wound healing activities.

Key words: C. quadrangularis, C. halicacabum, D. viscosa, GC-MS and FT-IR, Bone healing

Article Info: Received 03 March, 2018; Review Completed 03 April 2018; Accepted 14 April 2018; Available online 15 May 2018

口: Cite this article as:

Ansarali S, Manikandan S, Lakshmanan GMA, Identification of biological components from potential bone healer medicinal plants, Journal of Drug Delivery and Therapeutics. 2018; 8(3):32-41

DOI: http://dx.doi.org/10.22270/jddt.v8i3.1762

*Address for Correspondence:

Ganapathy Murugan Alagu Lakshmanan, Department of Botany, Thiru Kolanjiappar Govt. Arts College, Vriddhachalam, India- 606001.

\section{INTRODUCTION}

Traditional Indian Medicine (TIM) is a noteworthy wellspring of possibly valuable new constituent for the improvement of chemotherapeutic specialists ${ }^{\mathbf{1}}$. It has been evaluated that natural prescriptions serve around $80 \%$ of the total worldwide population ${ }^{2}$. The utilization of plants in the administration and treatment of sicknesses and natural products are considered organically dynamic mixes. Cissus quadrangularis L, belong to the family Vitaceae and distributed in India, Sri Lanka, Malaysia, Thailand and Africa. The stem portion of $C$. quadrangularis is utilized for the treatment of skin contaminations, blockage, heaps, frailty, asthma, sporadic feminine cycle, consumes, wounds and antiulcer $^{3}$ anti-oxidant, hostile to malignancy activity ${ }^{4}$, microbial activities ${ }^{5}$, against osteoporotic activity ${ }^{6}$, weight $\operatorname{loss}^{7}$, ant-inflammatory ${ }^{8}$ and bone crack healing ${ }^{9}$. Cardiospermum halicacabum belong to with the family Sapindaceae is a climber. These two species are widely distributed in tropical and subtropical Asia and Africa ${ }^{\mathbf{1 0}}$. The entire plant has been used in the treatment of stiffness, lumbago, diuretic, stomachic, ear infection, cough, emetic, purgative and rheumatic. The seeds are utilized a diaphoretic ${ }^{11}$, anti-malarial ${ }^{\mathbf{1 2}}$, anti-oxidant and anti-inflammatory ${ }^{13,14}$. C. halicacabum roots have been utilized for the treatment of epilepsy and tension disorders ${ }^{15}$. D. viscosa is placed in the family Sapindaceae and distributed in India and Nepal. It is utilized for different therapeutic purposes such as diuretic, anti-inflammatory, anti-ulcer ${ }^{16-17}$, snake bites ${ }^{18}$ and anti-oxidant ${ }^{19}$.

This study is mainly focus on to compare the divergence of biological compounds identification investigated by GC-MS and FT-IR analyses. GC-MS investigation was made by a typical library search (NIST, WILEY) and literature comparison and the biological activities of these compounds were studied for possible drug development of active compounds. 


\section{MATERIALS AND METHODS}

\section{Collection and preparation of plant material}

The plants were collected from natural habitats and the herbarium specimen of the same was prepared and deposited in the Department of Botany, Annamalai University with the accession number for $C$. quadrangularis (AUBOT343), C. halicacabum (AUBOT347) and D. viscosa (AUBOT340). The plants were washed completely in running tap water to remove soil particles and the plant parts were separated and shade dried. The shade dried plant parts were separately and stored in air tight container for further analysis.

\section{Chemicals}

The chemicals were obtained from Himedia, Mumbai, India and the solvents used were of phytochemical analyses

\section{Equipment}

Equipment's used on this experiment consist of GC-MS and FT-IR. GC-MS was used for the identity of compounds in samples and FT-IR becomes used for identification of functional agencies provided within the species of $C$. quadrangularis, D. viscosa and $C$. halicacabum.

\section{Plant sample extraction}

Shade dried and powdered plant materials were successively extracted with methanol with gentle stirring for 72 hours. The extraction were passed through Whatmann No. 1 paper and collected.

\section{Gas Chromatography- Mass Spectrum Analysis (GC-MS)}

25 gm of the stem and leaves powder C. quadrangularis, C. halicacabum and D. viscosa were soaked in $95 \%$ methanol for 12 hours. The extracts were then filtered through Whatmann filter No. 1 along with 2 gm Sodium sulphate to remove the sediments and traces of water in the filtrate. Then the filtrate was concentrated by introducing bubbling nitrogen gas into the solution. The plant extract contains both polar and non-polar phytochemicals. $2 \mu \mathrm{l}$ of the plant methanolic extract filtrate was used for GC-MS analysis. GC-MS analysis of the methanolic extract of the plant samples taken for this study was performed by using a Perkin- Elmer GC clarus- 500 system comprising an $\mathrm{AOC} 20^{\circ} \mathrm{C}$ auto sampler and a Gas chromatograph intergraded to a mass spectrometer equipped with Elite - 5MS fused capillary column. Helium gas was used as a carrier gas at a constant flow rate of $1 \mathrm{ml} / \mathrm{min}$, and an injector where of $2 \mu \mathrm{l}$ was employed. The inject temperature was maintained at $250^{\circ} \mathrm{C}$, the ion source temperature was $200^{\circ} \mathrm{C}$ and oven temperature was programmed from $110^{\circ} \mathrm{C}$, with an increase of $10^{\circ} \mathrm{C} / \mathrm{min}$ to $200^{\circ} \mathrm{C}$, then $5^{\circ} \mathrm{C} / \mathrm{min} 280^{\circ} \mathrm{C}$ ending with a $9 \mathrm{~min}$ isothermal at $280^{\circ} \mathrm{C}$. The solvent delay was 0 to $2 \mathrm{~min}$, and the total GC/MS running time was $36 \mathrm{~min}$. The relative percentage amount of each component was calculated by comparing its average peak area to the total areas. The Mass detector used in this analysis was turbo- Mass gold Perkin- Elmer and the software adopted to handle Mass spectra and chromatogram was a Turbo - Mass Ver.5.2.

\section{FT-IR analysis}

The FT-IR analysis was done by utilizing Perkin Elmer Spectrum Version 10.03.09 framework, which was utilized to identify the practical gatherings of the compound. A little measure of concentrate was set specifically on the zinc solenoid piece and consistent weight. Information of infrared retentive, gathered over the wave number extended from $4000 \mathrm{~cm}^{-1}$ to $400 \mathrm{~cm}^{-1}$ utilizing spectra programming. Tests were keep running in triplicate and every one of them were embraced inside a day time span.

\section{RESULTS AND DISCUSSION}

\section{GC-MS}

The phytochemical constituents present in the stem methanolic extract of $C$. quadrangularis showed fifty four constituents. Out of these fifty four constituents five constituents were majorly present such as 3-O-MethylD-glucose, D-Allose, 9,12,15-Octa decatrienoic acid, Phytol and Pentadecanoic Acid. Based on GC-MS spectrum confirmed the compounds with retention time $23.574,19.237,28.706,25.037$ and 26.250 respectively (Figure 1). Apart from the above mentioned compounds, the 3-O-Methyl-D-glucose was containing the percentage of 51.85 and it was identified as active compound of the species $C$. quadrangularis. The molecular formula of the compound was $\mathrm{C}_{7} \mathrm{H}_{14} \mathrm{O}_{6}$ (Table 1).

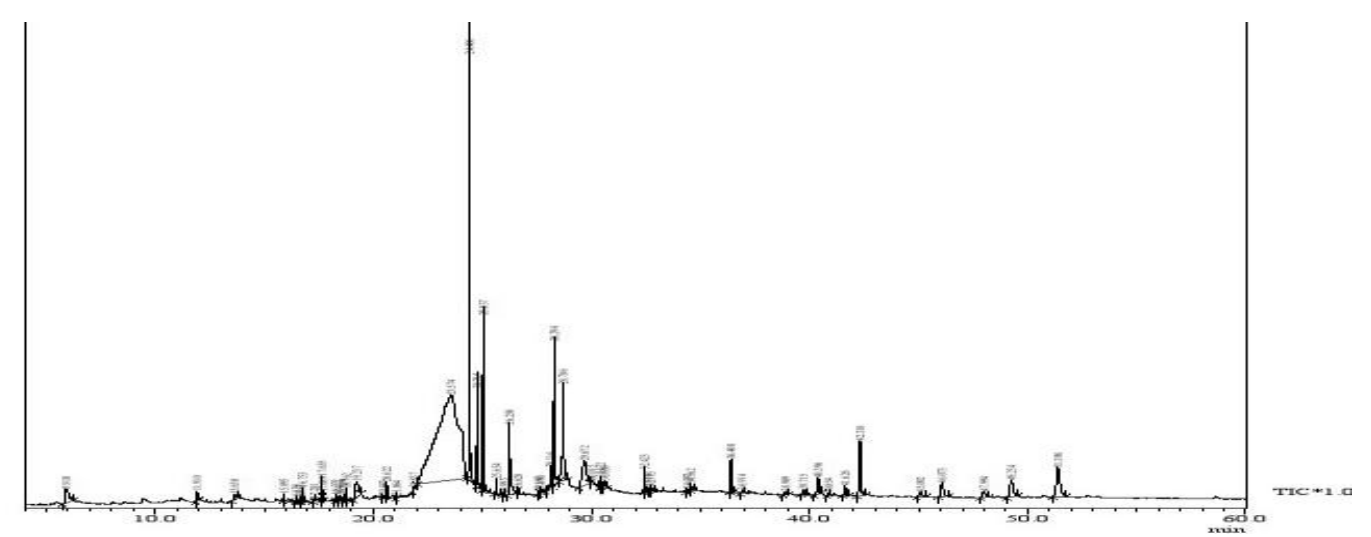

Figure 1: GC-MS Chromatogram of methanolic Stem extract of C. quadrangularis 
Table 1: GC-MS report of methanolic Stem extract of $C$. quadrangularis

\begin{tabular}{|c|c|c|c|c|c|}
\hline Peak & $\begin{array}{c}\text { R. } \\
\text { Time }\end{array}$ & Area\% & Name of the compounds & $\begin{array}{l}\text { Molecular } \\
\text { formula }\end{array}$ & $\begin{array}{l}\text { Molecular } \\
\text { weight }\end{array}$ \\
\hline 1 & 5.910 & 1.48 & 1H-Pyrrole & $\mathrm{C}_{4} \mathrm{H}_{5} \mathrm{~N}$ & 67 \\
\hline 2 & 11.910 & 0.32 & 2,3-Dihydro-3,5-Dihydroxy-6-Methyl-4h-Pyran-One & $\mathrm{C}_{6} \mathrm{H}_{8} \mathrm{O}_{4}$ & 144 \\
\hline 3 & 13.630 & 0.31 & 2h-Pyran-2-One, 5,6-Dihydro-6-Pentyl- & $\mathrm{C}_{10} \mathrm{H}_{16} \mathrm{O}_{2}$ & 168 \\
\hline 4 & 15.895 & 0.12 & 2.Alpha.,7,8-trimethylacenaphthylene & $\mathrm{C}_{15} \mathrm{H}_{24}$ & 204 \\
\hline 5 & 16.439 & 0.04 & $\begin{array}{l}\text { 2-(3-Isopropyl-4-Methyl-3-Penten-1-Ynyl)-2- } \\
\text { Methycyclobutanone }\end{array}$ & $\mathrm{C}_{14} \mathrm{H}_{20} \mathrm{O}$ & 204 \\
\hline 6 & 16.663 & 0.04 & 3,6,6,7-Tetramethyl-3-Vinyl-2,3,3a,4,5,6-Hexahydro-1h-Indene & $\mathrm{C}_{15} \mathrm{H}_{24}$ & 204 \\
\hline 7 & 16.753 & 0.25 & $\begin{array}{l}\text { Tricyclo[4.4.0.0(2,7)]Dec-3-Ene, 1,3-Di Methyl -8-(1- } \\
\text { Methylethyl) }\end{array}$ & $\mathrm{C}_{15} \mathrm{H}_{24}$ & 204 \\
\hline 8 & 17.281 & 0.04 & Aromadenrene & $\mathrm{C}_{15} \mathrm{H}_{24}$ & 204 \\
\hline 9 & 17.635 & 0.36 & Trans (.beta.)-caryophyllene & $\mathrm{C}_{15} \mathrm{H}_{24}$ & 204 \\
\hline 10 & 17.689 & 0.07 & Isoledene & $\mathrm{C}_{15} \mathrm{H}_{24}$ & 204 \\
\hline 11 & 18.272 & 0.11 & 1,4,8-Cycloundecatriene ,2,6,6,9-Tetra Methyl & $\mathrm{C}_{15} \mathrm{H}_{24}$ & 204 \\
\hline 12 & 18.412 & 0.09 & 1H-Cycloprop [E]Azulene, & $\mathrm{C}_{15} \mathrm{H}_{24}$ & 204 \\
\hline 13 & 18.634 & 0.10 & 6.Alpha.-Cadina-4,9-Diene, (-)- & $\mathrm{C}_{15} \mathrm{H}_{24}$ & 204 \\
\hline 14 & 18.762 & 0.20 & Beta.-ylangene & $\mathrm{C}_{15} \mathrm{H}_{24}$ & 204 \\
\hline 15 & 19.237 & 9.28 & D-Allose & $\mathrm{C}_{6} \mathrm{H}_{12} \mathrm{O}_{6}$ & 180 \\
\hline 16 & 20.443 & 0.03 & 1-Tridecanol & $\mathrm{C}_{13} \mathrm{H}_{28} \mathrm{O}$ & 200 \\
\hline 17 & 20.622 & 0.31 & (-)-5-Oxatricyclo [8.2.0.0(4,6)]Dodecane,, & $\mathrm{C}_{15} \mathrm{H}_{24} \mathrm{O}$ & 220 \\
\hline 18 & 21.064 & 0.05 & Humulene Oxide & $\mathrm{C}_{15} \mathrm{H}_{24} \mathrm{O}$ & 220 \\
\hline 19 & 21.837 & 0.07 & $\begin{array}{l}\text { (1ar-(1aalpha, 5abeta, 9ar(*)))-5a,9,9- } \\
\text { Trimethyloctahydrobenzo(D)Cycloprop(C)Oxepin-2,4 }\end{array}$ & $\mathrm{C}_{14} \mathrm{H}_{20} \mathrm{O}_{3}$ & 236 \\
\hline 20 & 23.574 & 51.85 & 3-O-Methyl-d-glucose & $\mathrm{C}_{7} \mathrm{H}_{14} \mathrm{O}_{6}$ & 194 \\
\hline 21 & 24.406 & 1.55 & 2,6,10-Trimethyl,14-Ethylene-14-Pentadecne & $\mathrm{C}_{20} \mathrm{H}_{38}$ & 278 \\
\hline 22 & 24.765 & 2.15 & Neophytadiene & $\mathrm{C}_{20} \mathrm{H}_{38}$ & 278 \\
\hline 23 & 25.037 & 3.41 & 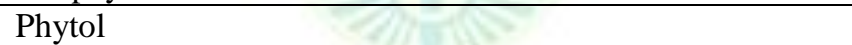 & $\mathrm{C}_{20} \mathrm{H}_{40} \mathrm{O}$ & 296 \\
\hline 24 & 25.654 & 0.37 & Hexadecanoic Acid, & $\mathrm{C}_{17} \mathrm{H}_{34} \mathrm{O}_{2}$ & 270 \\
\hline 25 & 25.993 & 0.05 & Isophytol & $\mathrm{C}_{20} \mathrm{H}_{40} \mathrm{O}$ & 296 \\
\hline 26 & 26.250 & 3.39 & Pentadecanoic Acid & $\mathrm{C}_{15} \mathrm{H}_{30} \mathrm{O}_{2}$ & 242 \\
\hline 27 & 26.628 & 0.08 & 14B-Pregnane & $\mathrm{C}_{21} \mathrm{H}_{36}$ & 288 \\
\hline 28 & 27.597 & 0.05 & Methyl 4-o-benzyl-.alpha.-1-rhamnopyra noside & $\mathrm{C}_{14} \mathrm{H}_{20} \mathrm{O}_{5}$ & 268 \\
\hline 29 & 27.690 & 0.04 & 1H-Indene, 3-Methyl- & $\mathrm{C}_{10} \mathrm{H}_{10}$ & 130 \\
\hline 30 & 28.116 & 0.63 & 9,12,15-Octadecatrie noic Acid, (Z,Z,Z)- & $\mathrm{C}_{19} \mathrm{H}_{32} \mathrm{O}_{2}$ & 292 \\
\hline 31 & 28.294 & 2.72 & 3,7,11,15-Tetramethyl-, [R-[R*,R*-(E)]] & $\mathrm{C}_{20} \mathrm{H}_{40} \mathrm{O}$ & 296 \\
\hline 32 & 28.706 & 3.83 & 9,12,15-Octadecatrienoic Acid, & $\mathrm{C}_{18} \mathrm{H}_{30} \mathrm{O}_{2}$ & 278 \\
\hline 33 & 29.672 & 2.93 & Cyclohexanol, 4-[(Tri Methylsilyl) Oxy] -, Cis- & $\mathrm{C}_{9} \mathrm{H}_{20} \mathrm{O}_{2}$ & 188 \\
\hline 34 & 30.015 & 0.25 & Decanoic Acid & $\mathrm{C}_{10} \mathrm{H}_{20} \mathrm{O}_{2}$ & 172 \\
\hline 35 & 30.422 & 0.20 & Octanoic acid, 2-di methylaminoethyl ester & $\mathrm{C}_{12} \mathrm{H}_{25} \mathrm{NO}_{2}$ & 215 \\
\hline 36 & 30.630 & 0.33 & 5-Phenyl-Pentanoic Acid, Ethyl Ester & $\mathrm{C}_{17} \mathrm{H}_{24} \mathrm{O}_{5}$ & 308 \\
\hline 37 & 32.423 & 0.42 & Fumaric acid, 2-di methyl aminoethylnonyl ester & $\mathrm{C}_{17} \mathrm{H}_{31} \mathrm{NO}_{4}$ & 313 \\
\hline 38 & 32.610 & 0.06 & 3-Cyclopentylpropionic Acid, & $\mathrm{C}_{12} \mathrm{H}_{23} \mathrm{NO}_{2}$ & 213 \\
\hline 39 & 32.715 & 0.13 & 2-Ethylbutyric Acid, Eicosyl Ester & $\mathrm{C}_{26} \mathrm{H}_{52} \mathrm{O}_{2}$ & 396 \\
\hline 40 & 34.397 & 0.10 & 1-Penten-3-One, 4-Methyl-1-Phenyl- & $\mathrm{C}_{12} \mathrm{H}_{14} \mathrm{O}$ & 174 \\
\hline 41 & 34.562 & 0.20 & Ethyl linolate & $\mathrm{C}_{19} \mathrm{H}_{32} \mathrm{O}_{2}$ & 292 \\
\hline 42 & 36.408 & 0.79 & Squalene & $\mathrm{C}_{30} \mathrm{H}_{50}$ & 410 \\
\hline 43 & 36.914 & 0.18 & TriacontylPentafluoropropionate & $\mathrm{C}_{33} \mathrm{H}_{61} \mathrm{~F}_{5} \mathrm{O}_{2}$ & 584 \\
\hline 44 & 38.909 & 0.15 & Chol-5-ene-3,24-diol & $\mathrm{C}_{24} \mathrm{H}_{40} \mathrm{O}_{2}$ & 360 \\
\hline 45 & 39.715 & 0.30 & Ergost-5-En-3-Ol, (3.Beta.)- & $\mathrm{C}_{28} \mathrm{H}_{48} \mathrm{O}$ & 400 \\
\hline 46 & 40.396 & 0.89 & Gamma.-tocopherol & $\mathrm{C}_{28} \mathrm{H}_{48} \mathrm{O}_{2}$ & 416 \\
\hline 47 & 40.854 & 0.10 & 5-chlorostigmastan-3-yl acetate & $\mathrm{C}_{31} \mathrm{H}_{53} \mathrm{C}_{1} \mathrm{O}_{2}$ & 492 \\
\hline 48 & 41.626 & 0.42 & Stigmast-5-En-3-Ol, (3.Beta.)- & $\mathrm{C}_{29} \mathrm{H}_{50} \mathrm{O}$ & 414 \\
\hline 49 & 42.318 & 2.70 & Vitamin E & $\mathrm{C}_{29} \mathrm{H}_{50} \mathrm{O}_{2}$ & 430 \\
\hline 50 & 45.092 & 0.40 & 24- Epicampesterol & $\mathrm{C}_{28} \mathrm{H}_{48} \mathrm{O}$ & 400 \\
\hline 51 & 46.073 & 1.05 & Stigmasta-5,22-Dien-3-ol & $\mathrm{C}_{29} \mathrm{H}_{48} \mathrm{O}$ & 412 \\
\hline 52 & 47.994 & 0.52 & Beta-di hydrofucosterol & $\mathrm{C}_{29} \mathrm{H}_{50} \mathrm{O}$ & 414 \\
\hline 53 & 49.254 & 1.50 & ,14,14a,14b-Octadbctadecahyro-2h-Picen-3-One & $\mathrm{C}_{30} \mathrm{H}_{48} \mathrm{O}$ & 424 \\
\hline \multirow[t]{2}{*}{54} & 51.398 & 2.98 & D:B-Friedo-B':A'-Neogammacer-5-En-3-one & $\mathrm{C}_{30} \mathrm{H}_{48} \mathrm{O}$ & 424 \\
\hline & & 100.00 & & & \\
\hline
\end{tabular}


GC-MS analysis of Methanolic extract of $C$. halicacabum leaves demonstrates nearness of forty two constituents. Out of these forty two constituents five constituents were majorly present such as Calix[4]arene, Epi-Psi-Taraxastanonol, Beta-Sitosterol, Tetradecanoic corrosive and Iso Longifolol with their respective retention time such as 43.196, 50.113, 48.078, 23.291 and 56.873 respectively (Figure 2). Based on the spectrum percentage area, Calix[4]arene (24.85) was identified as major active compound and the molecular formula was $\mathrm{C}_{28} \mathrm{H}_{24} \mathrm{O}_{4}$ (Table 2).

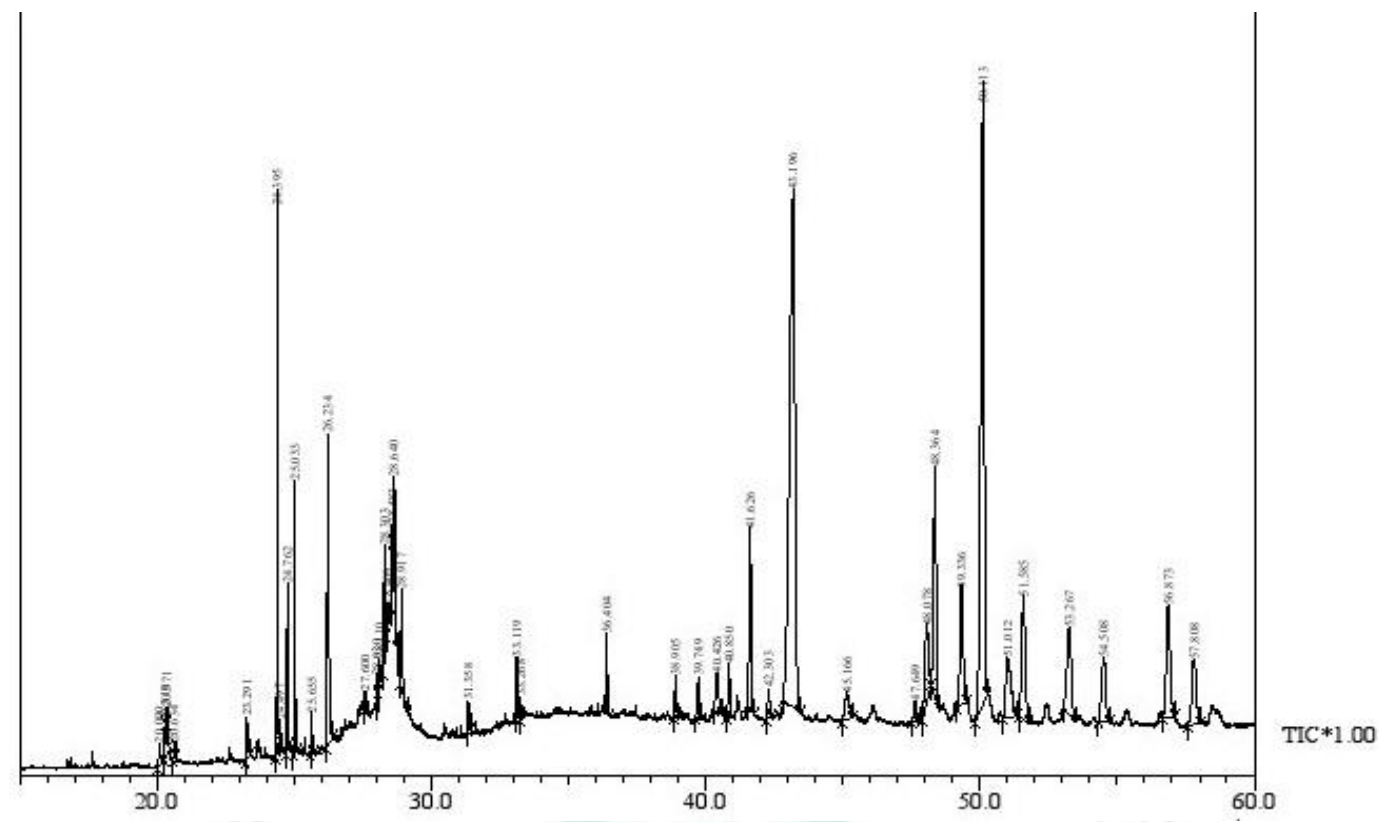

Figure 2: GC-MS Chromatogram of methanolic Leaf extract of C. halicacabum

Table 2: GC-MS report of methanolic Leaf extract of $C$. halicacabum

\begin{tabular}{|c|c|c|c|c|c|}
\hline Peak & $\begin{array}{c}\text { R. } \\
\text { Time }\end{array}$ & Area \% & Name of the compounds & $\begin{array}{c}\text { Molecular } \\
\text { formula }\end{array}$ & M.W. \\
\hline 1 & 20.090 & 0.25 & Dodecanoic acid & $\mathrm{C}_{12} \mathrm{H}_{24} \mathrm{O}_{2}$ & 200 \\
\hline 2 & 20.310 & 0.15 & Glutaric acid, 2-(4-fluorophenyl)ethyl 2-methylhex-3-yl ester & $\mathrm{C}_{20} \mathrm{H}_{29} \mathrm{FO}_{4}$ & 352 \\
\hline 3 & 20.371 & 0.15 & 1,2-Benzenedicarboxy lic acid,bis(2-methyl propyl) ester & $\mathrm{C}_{16} \mathrm{H}_{22} \mathrm{O}_{4}$ & 278 \\
\hline 4 & 20.634 & 0.12 & 1,2-Benzenedicarboxy lic acid, diethyl ester & $\mathrm{C}_{12} \mathrm{H}_{14} \mathrm{O}_{4}$ & 222 \\
\hline 5 & 23.291 & 3.88 & tetradecanoic acid & $\mathrm{C}_{14} \mathrm{H}_{28} \mathrm{O}_{2}$ & 228 \\
\hline 6 & 24.395 & 0.49 & 2,6,10-Trimethyl,14-ethylene-14-pentadecne & $\mathrm{C}_{20} \mathrm{H}_{38}$ & 278 \\
\hline 7 & 24.497 & 0.17 & 2-Pentadecanon, 6,10,14-trimethyl- & $\mathrm{C}_{18} \mathrm{H}_{36} \mathrm{O}$ & 268 \\
\hline 8 & 24.762 & 1.18 & Neophytadine & $\mathrm{C}_{20} \mathrm{H}_{38}$ & 278 \\
\hline 9 & 25.033 & 1.95 & 3,7,11,15-Tetramethyl-2-hexadecen-1-ol,3,7,11,15-tetramethyl & $\mathrm{C}_{20} \mathrm{H}_{40} \mathrm{O}$ & 296 \\
\hline 10 & 25.655 & 0.31 & Hexadecanoic acid, methyl ester & $\mathrm{C}_{17} \mathrm{H}_{34} \mathrm{O}_{2}$ & 270 \\
\hline 11 & 26.234 & 3.76 & Pentadecanoic acid & $\mathrm{C}_{15} \mathrm{H}_{30} \mathrm{O}_{2}$ & 242 \\
\hline 12 & 27.600 & 0.21 & 9-Octadecenoic acid (z) & $\mathrm{C}_{18} \mathrm{H}_{34} \mathrm{O}_{2}$ & 282 \\
\hline 13 & 28.020 & 0.15 & 9,12-Octadecadienoic acid, methyl ester & $\mathrm{C}_{19} \mathrm{H}_{34} \mathrm{O}_{2}$ & 294 \\
\hline 14 & 28.110 & 0.25 & 9,12,15-Octadecatrienoic acid, methyl ester, $(\mathrm{z}, \mathrm{z}, \mathrm{z})$ - & $\mathrm{C}_{19} \mathrm{H}_{32} \mathrm{O}_{2}$ & 292 \\
\hline 15 & 28.303 & 1.02 & Phytol isomer & $\mathrm{C}_{20} \mathrm{H}_{40} \mathrm{O}$ & 296 \\
\hline 16 & 28.409 & 0.21 & Tetradecanoic acid, methyl ester & $\mathrm{C}_{15} \mathrm{H}_{30} \mathrm{O}_{2}$ & 242 \\
\hline 17 & 28.583 & 0.29 & 9,12-Octadecadienoic acid (z,z)- & $\mathrm{C}_{18} \mathrm{H}_{32} \mathrm{O}_{2}$ & 280 \\
\hline 18 & 28.640 & 1.49 & Cis-vaccenic acid & $\mathrm{C}_{18} \mathrm{H}_{34} \mathrm{O}_{2}$ & 282 \\
\hline 19 & 28.917 & 1.02 & Octadecanoic acid & $\mathrm{C}_{18} \mathrm{H}_{36} \mathrm{O}_{2}$ & 284 \\
\hline 20 & 31.358 & 0.32 & Eicosanoic acid & $\mathrm{C}_{20} \mathrm{H}_{40} \mathrm{O}_{2}$ & 312 \\
\hline 21 & 33.119 & 0.55 & 1,2-Benzene di carboxylic acid & $\mathrm{C}_{24} \mathrm{H}_{38} \mathrm{O}_{4}$ & 390 \\
\hline 22 & 33.268 & 0.17 & Bis(2- Ethylhexyl) Phthalate & $\mathrm{C}_{24} \mathrm{H}_{38} \mathrm{O}_{4}$ & 390 \\
\hline 23 & 36.404 & 0.68 & Squalene & $\mathrm{C}_{30} \mathrm{H}_{50}$ & 410 \\
\hline 24 & 38.905 & 0.53 & Beta-sitosterin & $\mathrm{C}_{29} \mathrm{H}_{50} \mathrm{O}$ & 414 \\
\hline 25 & 39.749 & 0.72 & 24-Epicampesterol & $\mathrm{C}_{28} \mathrm{H}_{48} \mathrm{O}$ & 400 \\
\hline 26 & 40.426 & 0.97 & Gamma.-tocopherol & $\mathrm{C}_{28} \mathrm{H}_{48} \mathrm{O}_{2}$ & 416 \\
\hline 27 & 40.850 & 0.81 & Fucosterol, beta- dihydro & $\mathrm{C}_{29} \mathrm{H}_{50} \mathrm{O}$ & 414 \\
\hline
\end{tabular}




\begin{tabular}{|l|l|l|l|l|l|}
\hline 28 & & & $\mathrm{C}_{47} \mathrm{H}_{82} \mathrm{O}_{2}$ & 678 \\
& 41.626 & 3.10 & Stigmast-5-en-3-ol, oleate & & \\
\hline 29 & 42.303 & 0.56 & Vitamin E & $\mathrm{C}_{29} \mathrm{H}_{50} \mathrm{O}_{2}$ & 430 \\
\hline 30 & 43.196 & 24.85 & Calix[4]arene & $\mathrm{C}_{28} \mathrm{H}_{24} \mathrm{O}_{4}$ & 424 \\
\hline 31 & 45.166 & 0.96 & Ergost-5-en-3-ol, (3.beta.,24r)- & $\mathrm{C}_{28} \mathrm{H}_{48} \mathrm{O}$ & 400 \\
\hline 32 & 47.649 & 0.49 & D-Friedoolean-14-en-3-one & $\mathrm{C}_{30} \mathrm{H}_{48} \mathrm{O}$ & 424 \\
\hline 33 & 48.078 & 5.68 & Beta-Sitosterol & $\mathrm{C}_{29} \mathrm{H}_{50} \mathrm{O}$ & 414 \\
\hline 34 & 48.364 & 2.66 & $4,4,6 a, 6 b, 8 a, 11,11,14 b-O c t a m e t h y l-1,4,4 a, 5,6,6 a, 6 b$ & $\mathrm{C}_{30} \mathrm{H}_{48} \mathrm{O}$ & 424 \\
\hline 35 & 49.336 & 3.24 & tetramethyltricyclo[5.4.0.0(4,11)] & $\mathrm{C}_{16} \mathrm{H}_{28} \mathrm{O}$ & 236 \\
\hline 36 & 50.113 & 18.24 & Epi-.Psi.- Taraxastanonol & $\mathrm{C}_{30} \mathrm{H}_{50} \mathrm{O}_{2}$ & 442 \\
\hline 37 & 51.012 & 2.67 & Methyl commate D & $\mathrm{C}_{31} \mathrm{H}_{50} \mathrm{O}_{4}$ & 486 \\
\hline 38 & 51.585 & 3.86 & D:A-Friedoolean-6-ene & $\mathrm{C}_{30} \mathrm{H}_{50}$ & 410 \\
\hline 39 & 53.267 & 3.21 & Stigmast-4-en-3-one & $\mathrm{C}_{29} \mathrm{H}_{48} \mathrm{O}$ & 412 \\
\hline 40 & 54.508 & 2.30 & Dimethyl\{bis[(4,8,8-trimethyldecahydro-1,4 methanoazulen-9-y) & $\mathrm{C}_{32} \mathrm{H}_{56} \mathrm{O}_{2}$ & 500 \\
\hline 41 & 56.873 & 3.91 & IsoLongifolol & $\mathrm{C}_{15} \mathrm{H}_{26} \mathrm{O}$ & 222 \\
\hline & & 100.00 & & \multicolumn{2}{|l}{} \\
\hline
\end{tabular}

Methanolic leaves extract of D. viscosa indicates fifty two constituents. There are five major constituents were present likely 7-methanoazulen-6-ol, Alpha-Methyl gluco furanoside, Pregn-4-Ene-3, 20-Dione, Palmitic acid and Alpha-Ionol. Based on GC-MS spectrum confirmed the compounds with retention time 39.058, $27.459,35.870,37.685$ and 26.577 respectively (Figure
3). Apart from the above mentioned compounds, the 7methanoazulen-6-ol, Alpha-Methyl gluco furanoside, Pregn-4-Ene-3, 20-Dione was containing the percentage of 28.32, 20.00 and 10.54. The 7-methanoazulen-6-ol was identified as active compound of the species $D$. viscosa. The molecular formula of the compound was $\mathrm{C}_{15} \mathrm{H}_{26} \mathrm{O}$ (Table 3).

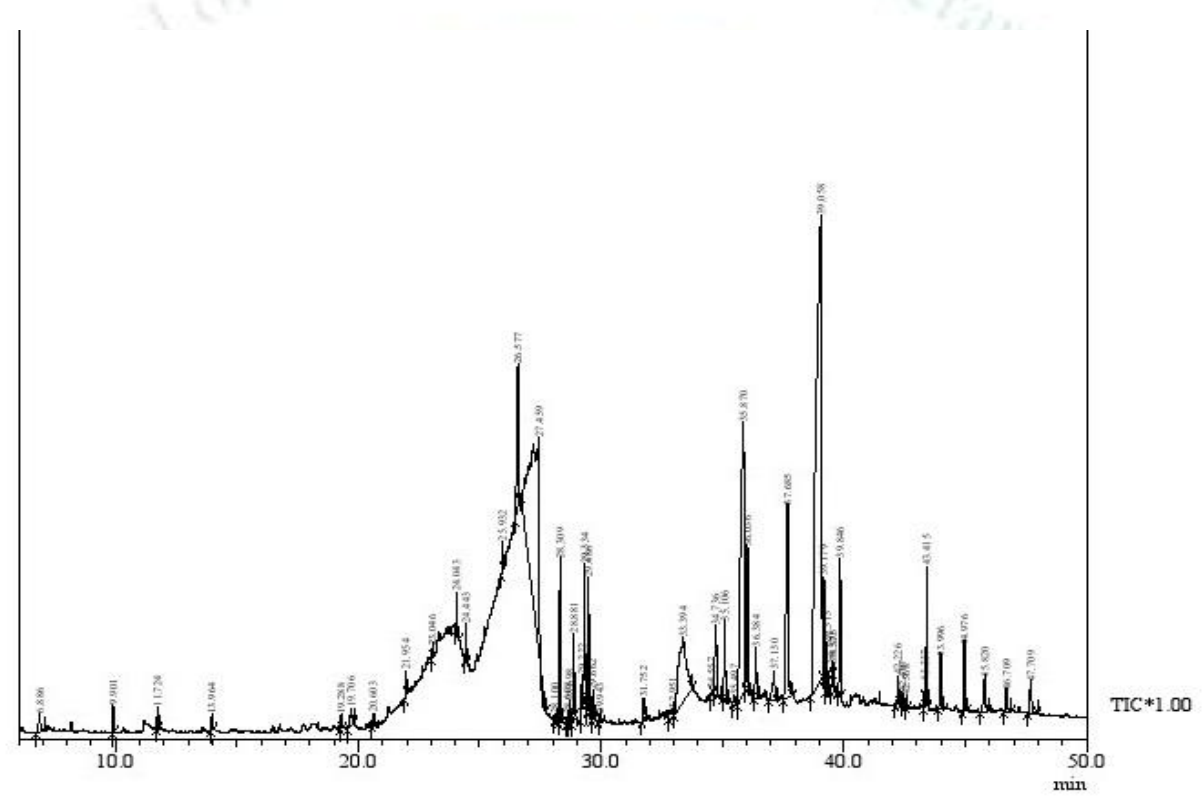

Figure 3: GC-MS Chromatogram of methanolic Leaf extract of D.viscosa

Table 3: GC-MS report of methanolic Leaf extract of $D$. viscosa

\begin{tabular}{|c|c|c|c|c|c|}
\hline Peak & $\begin{array}{l}\text { R. } \\
\text { Time }\end{array}$ & Area\% & Name of the Compounds & $\begin{array}{l}\text { Molecular } \\
\text { Formula } \\
\end{array}$ & M.W. \\
\hline 1 & 6.886 & 0.36 & Monomethylmalonate & $\mathrm{C}_{4} \mathrm{H}_{6} \mathrm{O}_{4}$ & 118 \\
\hline 2 & 9.901 & 0.28 & 4H-Pyran-4-one,2,3-dihydro-3,5-dihydroxy-6-methyl- & $\mathrm{C}_{6} \mathrm{H}_{8} \mathrm{O}_{4}$ & 144 \\
\hline 3 & 11.724 & 0.22 & 2,3-Dihydro-Benzofuran & $\mathrm{C}_{8} \mathrm{H}_{8} \mathrm{O}$ & 120 \\
\hline 4 & 13.964 & 0.15 & 2-Methoxy-4-Vinylphenol & $\mathrm{C}_{9} \mathrm{H}_{10} \mathrm{O}_{2}$ & 150 \\
\hline 5 & 19.288 & 0.14 & 3',5'-Dimethoxyacetophenone & $\mathrm{C}_{10} \mathrm{H}_{12} \mathrm{O}_{3}$ & 180 \\
\hline 6 & 19.706 & 0.43 & 2,3-Di-O-methyl-D-xylopyranose & $\mathrm{C}_{7} \mathrm{H}_{14} \mathrm{O}_{5}$ & 178 \\
\hline 7 & 20.603 & 0.14 & Megastigmatrienone & $\mathrm{C}_{13} \mathrm{H}_{18} \mathrm{O}$ & 190 \\
\hline 8 & 21.954 & 0.28 & 2H-1-Benzopyran-5-ol, 3,4-Dihydro-2,2-dimethyl- & $\mathrm{C}_{11} \mathrm{H}_{14} \mathrm{O}_{2}$ & 178 \\
\hline 9 & 23.046 & 0.09 & Tetradecanoic acid & $\mathrm{C}_{14} \mathrm{H}_{28} \mathrm{O}_{2}$ & 228 \\
\hline 10 & 24.043 & 0.47 & 2,4,6-Cycloheptatrien-1-one, 2-Hydroxy-5-(3-Methyl-2-But & $\mathrm{C}_{15} \mathrm{H}_{18} \mathrm{O}_{2}$ & 230 \\
\hline
\end{tabular}




\begin{tabular}{|c|c|c|c|c|c|}
\hline 11 & 24.443 & 0.31 & 2,6,10-Trimethyl,14-Ethylene-14-Pentadecne & $\mathrm{C}_{20} \mathrm{H}_{38}$ & 278 \\
\hline 12 & 25.932 & 0.27 & Hexadecanoic Acid, Methyl Ester & $\mathrm{C}_{17} \mathrm{H}_{34} \mathrm{O}_{2}$ & 270 \\
\hline 13 & 26.577 & 5.91 & Palmitic acid & $\mathrm{C}_{16} \mathrm{H}_{32} \mathrm{O}_{2}$ & 256 \\
\hline 14 & 27.459 & 20.00 & Alpha-Methylglucofuranoside & $\mathrm{C}_{7} \mathrm{H}_{14} \mathrm{O}_{6}$ & 194 \\
\hline 15 & 28.100 & 0.05 & Heptadecamonoenoic Acid & $\mathrm{C}_{17} \mathrm{H}_{32} \mathrm{O}_{2}$ & 268 \\
\hline 16 & 28.309 & 1.67 & Diallylphenylvinylsilane & $\mathrm{C}_{14} \mathrm{H}_{18}$ & 214 \\
\hline 17 & 28.608 & 0.08 & 9,12-Octadecadienoic acid (Z,Z)-, methyl ester & $\mathrm{C}_{19} \mathrm{H}_{34} \mathrm{O}_{2}$ & 294 \\
\hline 18 & 28.698 & 0.24 & 9,12,15-Octadecatrienoic acid, methyl ester, (Z,Z,Z)- & $\mathrm{C}_{19} \mathrm{H}_{32} \mathrm{O}_{2}$ & 292 \\
\hline 19 & 28.881 & 0.89 & Phytol & $\mathrm{C}_{20} \mathrm{H}_{40} \mathrm{O}$ & 296 \\
\hline 20 & 29.222 & 0.44 & 9,12-Octadecadienoic acid (Z,Z)- & $\mathrm{C}_{18} \mathrm{H}_{32} \mathrm{O}_{2}$ & 280 \\
\hline 21 & 29.334 & 1.63 & Dichloroacetic acid, tridec-2-ynyl ester & $\mathrm{C}_{15} \mathrm{H}_{24} \mathrm{C}_{12} \mathrm{O}_{2}$ & 306 \\
\hline 22 & 29.486 & 1.45 & $\begin{array}{l}\text { (3aS,4R,7R)-1,4,9,9-Tetramethyl-5,6,7,8-tetrahydro-4H-3a,7- } \\
\text { methanoazulene }\end{array}$ & $\mathrm{C}_{15} \mathrm{H}_{22}$ & 202 \\
\hline 23 & 29.662 & 0.29 & Octadecanoic acid & $\mathrm{C}_{18} \mathrm{H}_{36} \mathrm{O}_{2}$ & 284 \\
\hline 24 & 29.943 & 0.08 & $\begin{array}{l}\text { (1R,4aR,4bS,7S,10aR)-1,4a,7-Trimethyl-7-vinyl- } \\
1,2,3,4,4 \mathrm{a}, 4 \mathrm{~b}, 5,6,7,8,10,10 \mathrm{a}-\end{array}$ & $\mathrm{C}_{20} \mathrm{H}_{30} \mathrm{O}$ & 286 \\
\hline 25 & 31.752 & 0.41 & Lup-20(29)-Ene-3,28-Diol, (3.Beta.)- & $\mathrm{C}_{30} \mathrm{H}_{50} \mathrm{O}_{2}$ & 442 \\
\hline 26 & 32.951 & 0.23 & Benzene, Hexaethyl- & $\mathrm{C}_{18} \mathrm{H}_{30}$ & 246 \\
\hline 27 & 33.394 & 2.49 & $\begin{array}{l}\text { Cycloprop[e]indene-1a,2(1H)-dicarboxaldehyde, 3a,4,5,6,6a,6b- } \\
\text { hexahydro-5,5,6b- }\end{array}$ & $\mathrm{C}_{15} \mathrm{H}_{20} \mathrm{O}_{2}$ & 232 \\
\hline 28 & 34.557 & 0.06 & $\begin{array}{l}\text { Cyclopropanecarboxylic acid, 2-[4-(1,1-dimethylethyl)phenyl]-, } \\
\text { ethyl ester- }\end{array}$ & $\mathrm{C}_{16} \mathrm{H}_{22} \mathrm{O}_{2}$ & 246 \\
\hline 29 & 34.736 & 1.87 & $\begin{array}{l}\begin{array}{l}\text { Pregn-5-en-20-one, } \\
\text { (3.beta.,16.alpha.)- }\end{array} \\
\text { 3-(acetyloxy)-16,17-epoxy-6-methyl-, } \\
\end{array}$ & $\mathrm{C}_{24} \mathrm{H}_{34} \mathrm{O}_{4}$ & 386 \\
\hline 30 & 35.106 & 1.15 & 4,5-Secocholest-6-en-4-oic acid, 5-oxo- & $\mathrm{C}_{27} \mathrm{H}_{44} \mathrm{O}_{3}$ & 416 \\
\hline 31 & 35.497 & 0.16 & 5-Isopropylidene-6-methyldeca-3,6,9-trien-2-one & $\mathrm{C}_{14} \mathrm{H}_{20} \mathrm{O}$ & 204 \\
\hline 32 & 35.870 & 10.54 & Pregn-4-Ene-3,20-Dione, 14-Hydroxy- & $\mathrm{C}_{21} \mathrm{H}_{30} \mathrm{O}_{3}$ & 330 \\
\hline 33 & 36.036 & 2.26 & Costunolide & $\mathrm{C}_{15} \mathrm{H}_{20} \mathrm{O}_{2}$ & 232 \\
\hline 34 & 36.384 & 0.88 & Bicyclo[5.3.0]decane, 2-methylene-5-(1-methylvinyl)-8-methyl- & $\mathrm{C}_{15} \mathrm{H}_{24}$ & 204 \\
\hline 35 & 37.130 & 0.87 & $\begin{array}{l}\begin{array}{l}\text { 2(1H)-Naphthalenone, } \\
\text { (Hydroxymethyl) }\end{array} \\
\end{array}$ & $\mathrm{C}_{15} \mathrm{H}_{22} \mathrm{O}_{2}$ & 234 \\
\hline 36 & 37.685 & 4.87 & Alpha-Ionol & $\mathrm{C}_{13} \mathrm{H}_{22} \mathrm{O}$ & 194 \\
\hline 37 & 39.058 & 28.32 & 7-methanoazulen-6-ol & $\mathrm{C}_{15} \mathrm{H}_{26} \mathrm{O}$ & 222 \\
\hline 38 & 39.179 & 1.25 & $\begin{array}{l}\text { Decahydronaphtho[2,3-b]furan-2-one, } \\
\text { yl)ethylamino]methyl]- }\end{array}$ & $\mathrm{C}_{20} \mathrm{H}_{29} \mathrm{~N}_{3} \mathrm{O}_{2}$ & 343 \\
\hline 39 & 39.313 & 0.64 & $\begin{array}{l}\text { 4,14-Dimethyl-11-isopropyltricyclo[7.5.0.0(10,14)]tetradec-4- } \\
\text { en-8-one }\end{array}$ & $\mathrm{C}_{19} \mathrm{H}_{30} \mathrm{O}$ & 274 \\
\hline 40 & 39.520 & 0.16 & 5-(3-Buten-1-Ynyl)-2,2'-Bithienyl & $\mathrm{C}_{12} \mathrm{H}_{8} \mathrm{~S}_{2}$ & 216 \\
\hline 41 & 39.575 & 0.12 & 7-Methoxy-3-(4-pentylcyclohexyl)-1,2-dihydronaphthalene & $\mathrm{C}_{22} \mathrm{H}_{32} \mathrm{O}$ & 312 \\
\hline 42 & 39.846 & 1.76 & $\begin{array}{l}\text { Pregnan-20-one, } \\
\text { (3.beta.,5.alpha.,6.beta.)- }\end{array}$ & $\mathrm{C}_{22} \mathrm{H}_{36} \mathrm{O}_{3}$ & 348 \\
\hline 43 & 42.226 & 0.26 & Spiro(9,9'-Bis(2-Methyl-9H-Fluorene)) & $\mathrm{C}_{15} \mathrm{H}_{25} \mathrm{I}$ & 332 \\
\hline 44 & 42.407 & 0.10 & $\begin{array}{l}\text { (R)-6-Methoxy-2,8-dimethyl-2-((4R,8R)-4,8,12- } \\
\text { trimethyltridecyl)chroman }\end{array}$ & $\mathrm{C}_{27} \mathrm{H}_{20}$ & 344 \\
\hline 45 & 42.568 & 0.11 & .gamma.-Tocopherol & $\mathrm{C}_{28} \mathrm{H}_{48} \mathrm{O}_{2}$ & 416 \\
\hline 46 & 43.337 & 0.16 & Ethyl 5,8,11,14,17-icosapentaenoate & $\mathrm{C}_{22} \mathrm{H}_{34} \mathrm{O}_{2}$ & 330 \\
\hline 47 & 43.415 & 1.49 & Vitamin E & $\mathrm{C}_{29} \mathrm{H}_{50} \mathrm{O}_{2}$ & 430 \\
\hline 48 & 43.996 & 0.81 & $\begin{array}{l}\text { (3S,3aR,6R,8aS)-7,7-Dimethyl-8-methyleneoctahydro-1H-3a,6- } \\
\text { methanoazulene- }\end{array}$ & $\mathrm{C}_{15} \mathrm{H}_{22} \mathrm{O}$ & 218 \\
\hline 49 & 44.976 & 1.10 & Stigmasterol & $\mathrm{C}_{29} \mathrm{H}_{48} \mathrm{O}$ & 412 \\
\hline 50 & 45.820 & 0.85 & .gamma.-Sitosterol & $\mathrm{C}_{29} \mathrm{H}_{50} \mathrm{O}$ & 414 \\
\hline 51 & 46.709 & 0.44 & 25-Homo-24-Ketocholesterol & $\mathrm{C}_{28} \mathrm{H}_{46} \mathrm{O}_{2}$ & 414 \\
\hline \multirow[t]{2}{*}{52} & 47.709 & 0.77 & 2,2-Dimethylcholest-7-En-3-Ol & $\mathrm{C}_{29} \mathrm{H}_{50} \mathrm{O}$ & 414 \\
\hline & & 100.00 & & & \\
\hline
\end{tabular}

\section{FT-IR}

FT-IR range was utilized to distinguish the utilitarian gathering of the dynamic segments in view of the peak an incentive in the point of infrared radiation. The FTIR analysis results showed that the methanolic stem extract of $C$. quadrangularis having the presence of
Alcohol, Aldehyde, Iso cyanides, Alkane, Primary alcohol, Chloro constituent (Table 4), which shows major peaks at $3420.91,2924.27,2846.85,2076.74$, 1647.04, 1454.75, 1409.96, 1053.44, 1032.56, 1017.56, 718.75 and 666.26 respectively (Figure 4). The FT-IR analysis of methanolic leaves extract of $C$. halicacabum, 
the presence of Alcohol, Iso cyanides, Alkyl Compound, Alkane, Primary alcohol, Chloro constituent (Table 5). Constituent which shows major peaks at 3402.43, 2926.05, 2226.24, 2077.15, 1644.48, 1454.30, 1412.48, $1055.44, \quad 1033.07, \quad 1018.25,906.19$ and 655.71 respectively (Figure 5). The FT-IR analysis methanolic leaves extract of D. viscosa showed Alcohol, Aldehyde, Nitrite, Alkane, Primary alcohol (Table 6). Constituent which shows high peaks at 3336.61, 2835.87, 1650.80, 1451.41, 1263.33 and 1018.19 (Figure 6).

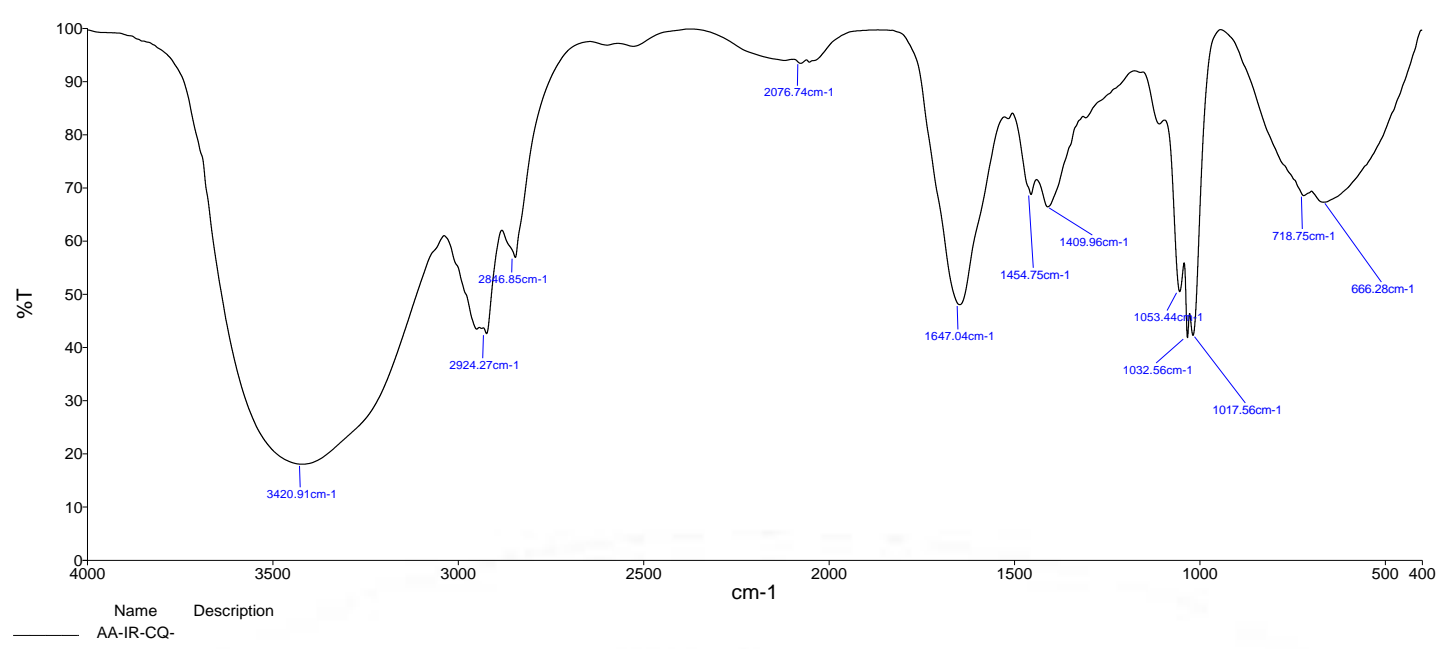

Figure 4: FT-IR Spectrum of methanolic Stem extract of C. quadrangularis

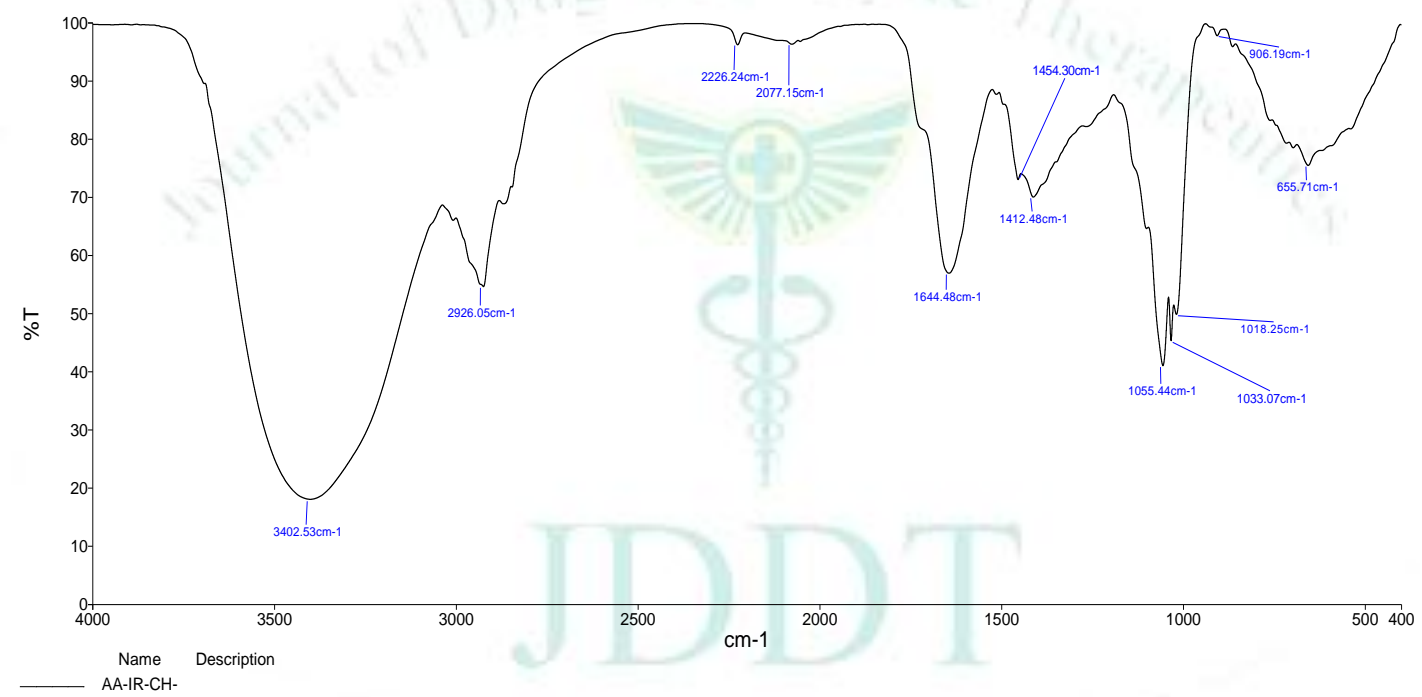

Figure 5: FT-IR Spectrum of methanolic Leaf extract of C. halicacabum

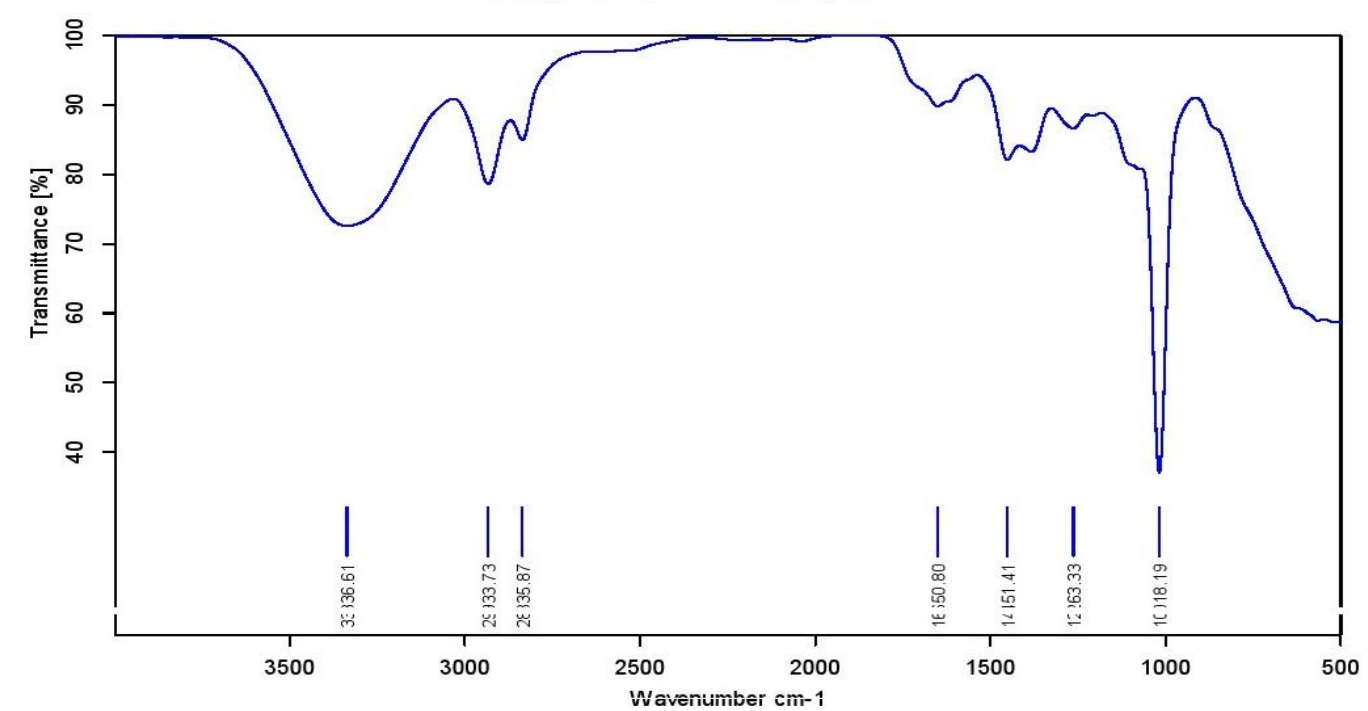

Figure 6: FT-IR Spectrum of methanolic Leaf extract of D.viscosa 
Table 4: FT-IR absorption and functional groups of Stem extract of $C$. quadrangularis

\begin{tabular}{|l|l|c|l|l|}
\hline S. No & Wave No. & Molecular Motion & Functional group & Absorption intensity \\
\hline 1 & $3420.91 \mathrm{~cm}-1$ & O-H & Alcohol & Strong \\
\hline 2 & $2924.27 \mathrm{~cm}-1$ & O-H & Alcohol & Medium \\
\hline 3 & $2846.85 \mathrm{~cm}-1$ & C=O & Aldehyde & Weak \\
\hline 4 & $2076.74 \mathrm{~cm}-1$ & C=N & Iso cyanides & Medium \\
\hline 5 & $1647.04 \mathrm{~cm}-1$ & O-NO & Nirite & Strong \\
\hline 6 & $1454.75 \mathrm{~cm}-1$ & C-H & Alkane & Medium \\
\hline 7 & $1409.96 \mathrm{~cm}-1$ & C-H & Alkane & Medium \\
\hline 8 & $1053.44 \mathrm{~cm}-1$ & C-O & Primary alcohol & Strong \\
\hline 9 & $1032.56 \mathrm{~cm}-1$ & C-O & Primary alcohol & Strong \\
\hline 10 & $1017.56 \mathrm{~cm}-1$ & C-O & Primary alcohol & Strong \\
\hline 11 & $718.75 \mathrm{~cm}-1$ & C-Cl & Chloro compound & Strong \\
\hline 12 & $666.26 \mathrm{~cm}-1$ & C-Cl & Chloro compound & Strong \\
\hline
\end{tabular}

Table 5: FT-IR absorption and functional groups of Leaf of $C$. halicacabum

\begin{tabular}{|l|l|c|l|l|}
\hline S. No & Wave No. & Molecular Motion & Functional group & Absorption intensity \\
\hline 1 & $3402.53 \mathrm{~cm}-1$ & O-H & Alcohol & Strong \\
\hline 2 & $2926.05 \mathrm{~cm}-1$ & O-H & Alcohol & Medium \\
\hline 3 & $2226.24 \mathrm{~cm}-1$ & $\mathrm{C}=\mathrm{N}$ & Iso cyanides & Medium \\
\hline 4 & $2077.15 \mathrm{~cm}-1$ & $\mathrm{C}=\mathrm{N}$ & Iso cyanides & Medium \\
\hline 5 & $1644.48 \mathrm{~cm}-1$ & $\mathrm{C}=\mathrm{N}$ & Alkyl compound & Strong \\
\hline 6 & $1454.30 \mathrm{~cm}-1$ & $\mathrm{C}-\mathrm{H}$ & Alkane & Medium \\
\hline 7 & $1412.48 \mathrm{~cm}-1$ & $\mathrm{C}-\mathrm{H}$ & Alkane & Medium \\
\hline 8 & $1055.44 \mathrm{~cm}-1$ & $\mathrm{C}-\mathrm{O}$ & Primary alcohol & Strong \\
\hline 9 & $1033.07 \mathrm{~cm}-1$ & $\mathrm{C}-\mathrm{O}$ & Primary alcohol & Strong \\
\hline 10 & $1018.25 \mathrm{~cm}-1$ & $\mathrm{C}-\mathrm{O}$ & Primary alcohol & Strong \\
\hline 11 & $906.19 \mathrm{~cm}-1$ & $\mathrm{C}-\mathrm{O}$ & Primary alcohol & Strong \\
\hline 12 & $655.71 \mathrm{~cm}-1$ & $\mathrm{C}-\mathrm{Cl}$ & Chloro compound & Strong \\
\hline
\end{tabular}

Table 6: FT-IR absorption and functional groups of Leaf of $D$. viscosa

\begin{tabular}{|l|l|c|l|l|}
\hline S. No & Wave No. & Molecular Motion & Functional group & Absorption intensity \\
\hline 1 & $3336.61 \mathrm{~cm}-1$ & O-H & Alcohol & Strong \\
\hline 2 & $2933.73 \mathrm{~cm}-1$ & O-H & Alcohol & Medium \\
\hline 3 & $2835.87 \mathrm{~cm}-1$ & C=O & Aldehyde & Weak \\
\hline 4 & $1650.80 \mathrm{~cm}-1$ & O-NO & Nitrite & Strong \\
\hline 5 & $1451.41 \mathrm{~cm}-1$ & C-H & Alkane & Medium \\
\hline 6 & $1263.33 \mathrm{~cm}-1$ & C-O & Alcohol & Medium \\
\hline 7 & $1018.19 \mathrm{~cm}-1$ & C-O & Primary alcohol & Strong \\
\hline
\end{tabular}

The biological therapeutic activities of identified major components of the stem extract of $C$. quadrangularis, leaves extracts of $C$. halicacabum and D. viscosa were given in Table 7. Therapeutic activities of these major components analysed by using Dr. Duke's Phytochemical and Ethno botanical data bases ${ }^{20}$

Table 7: Therapeutic activity of major compounds in C. quadrangularis, C. halicacabum and D. viscosa

\begin{tabular}{|l|l|l|l|}
\hline $\begin{array}{l}\text { S. } \\
\text { No }\end{array}$ & $\begin{array}{l}\text { Name of the } \\
\text { Compound }\end{array}$ & $\begin{array}{l}\text { Compound } \\
\text { Nature }\end{array}$ & Therapeutic activity \\
\hline 1 & 3-O-Methyl-d-glucose & Alcohol & $\begin{array}{l}\text { Osteoporosis, anaesthetics, anti-epileptics, anti-convulsants and } \\
\text { anti-parkinson drugs. }\end{array}$ \\
\hline 2 & D-Allose & Allose & $\begin{array}{l}\text { Treating wounds, ulcers, burns, scars, keloids, anti-pruritic, anti- } \\
\text { psoriatic, arthritis and carthorses. }\end{array}$ \\
\hline 3 & $\begin{array}{l}9,12,15-O c t a d e c a t r i e n o i c \\
\text { acid }\end{array}$ & Fatty acid & $\begin{array}{l}\text { Anti-psoriatic, joint disorders, anti-pyretic, anti-inflammatory, } \\
\text { wounds healing, anti-Tumor activities. }\end{array}$ \\
\hline 4 & Phytol & $\begin{array}{l}\text { Acyclicditerpene } \\
\text { alcohol }\end{array}$ & $\begin{array}{l}\text { Anti-tubercular activity, allergic disorders and anti-microbial } \\
\text { activities. }\end{array}$ \\
\hline 5 & Pentadecanoic Acid & Fatty acid & Anti-cancer drug, anti-asthmatics and anti-abortive. \\
\hline
\end{tabular}




\begin{tabular}{|l|l|l|l|}
\hline 6 & Calix[4]arene & Calixarene & Anti-cancer (cancer immunotherapy) \\
\hline 7 & Epi-Psi-Taraxastanonol & Terpenoids & Cardiovascular disease \\
\hline 8 & Beta-Sitosterol & Sterol & $\begin{array}{l}\text { Swelling, bladder infections, urinary tract infections, anti- } \\
\text { carcinogenic and anti-atherogenic properties, Anti-asthmatics } \\
\text { and throat disorders. }\end{array}$ \\
\hline 9 & Tetradecanoic acid & Fatty acid & $\begin{array}{l}\text { Antitumor activity, anti-spasmodic, anti-asthmatics and throat } \\
\text { disorders. }\end{array}$ \\
\hline 10 & IsoLongifolol & Ketone & Anti-microbial activities and anti-inflammatory \\
\hline 11 & 7 -methanoazulen-6-ol & Alcohol & Anti-perspirants and anti-pruritic \\
\hline 12 & $\begin{array}{l}\text { Alpha- } \\
\text { Methylglucofuranoside }\end{array}$ & Glycoside & $\begin{array}{l}\text { Treating bacterial and, fungal infections, anti-abortive, anti- } \\
\text { bacterial, anti-viral and allergic disorders. }\end{array}$ \\
\hline 13 & Pregn-4-Ene-3,20-Dione & Steroid & $\begin{array}{l}\text { Sexualdisorders,baldness, anti-psoriatic, anti-pyretic, anti- } \\
\text { inflammatory, Anti-bacterial and Anti-allergic. }\end{array}$ \\
\hline 14 & Palmitic acid & Fatty acid & $\begin{array}{l}\text { Throat disorders,anti-asthmatics, anti-pruritic, anti-psoriatic, } \\
\text { anti-epileptics, anti-convulsantsand anti-migraine. }\end{array}$ \\
\hline 15 & Alpha-Ionol & Alcohol & Anti-diabetics and anti-perspirants. \\
\hline
\end{tabular}

\section{Source: J. A. Duke (1992) Database of Biologically Active Phytochemicals ${ }^{20}$}

\section{CONCLUSION}

In the present study, the GC-MS analysis of the methanolic extract of $C$. quadrangularis, $C$. halicacabum and D. viscosa majorly present of fifteen compounds. Such as 3-O-Methyl-D-glucose, D-Allose, 9,12,15-Octadecatrienoic acid, Phytol, Pentadecanoic acid. Calix [4] arene, Epi-.Psi.- Taraxastanonol, BetaSitosterol,Tetradecanoic acid and Iso Longifolol,7methanoazulen-6-ol, Alpha-Methylglucofuranoside, Pregn-4-Ene-3, 20-Dione, Alpha-Ionol and Palmitic acid. Presence of these compounds were justifies,

\section{REFERENCES}

1. Ankita J, Jain A. Tridax Procumbens (L): A Weed with Immense Medicinal Importance: A Review. International Journal of Pharma and Bio Sciences, 2012; 3(1):544-552.

2. World Health Organization, General Guidelines for Methodologies on Research and Evaluation of Traditional Medicine Switzerland: Geneva 2001

3. Jaya C, Anuradha CV. Cissus quadrangularis stem alleviates insulin resistance, oxidative injury and fatty liver disease in rats fed high fat plus fructose diet. Food and Chemical Toxicology, 2010; 48(8-9):2021-2029.

4. Vijayalakshmi A, Kumar P. R, Sakthi Priyadarsini S, Meenaxshi C. In Vitro Antioxidant and Anticancer Activity of Flavonoid Fraction from the Aerial Parts of Cissus quadrangularis L. against Human Breast Carcinoma Cell Lines. Journal of Chemistry, 2013:1-9.

5. Marquis G. Ramasamy B. Banwarilal S. Munusamy AP. Evaluation of antibacterial activity of plant mediated $\mathrm{CaO}$ nanoparticles using Cissus quadrangularis extract. Journal of Photochemistry and Photobiology B: Biology, 2016; 155(6):28-33.

6. Bhagath K, Muddannarao S, Gopalan K, Nampurath R, Mallikarjuna R, Chamallamudi C, Prasad K. Evidence-based assessment of anti-osteoporotic activity of petroleum-ether extract of Cissus quadrangularis Linn. On ovariectomyinduced osteoporosis. Upsala Journal of Medical Science, 2009; 114(3):140-148.

7. Oben J, Kuate D, Agbor G, Momo C, Talla X. The use of a Cissus quadrangularis $\mathrm{L}$. formulation in the management of weight loss and metabolic syndrome. Lipids in Health and Disease 2006, 5:1-7.

8. Arti BM, Talmale S, Kumar N, Gupta G, Reddanna P, Samar $\mathrm{K}$, Das MB. Evaluation of Cissus quadrangularis extracts as an inhibitor of COX, 5-LOX, and pro-inflammatory mediators. Journal of Ethnopharmacology, 2012; 141(3):989996. wounds healing, arthritis, anti-inflammatory Antitubercular activity anti-microbial activities, antiasthmatics, throat disorders, Sexual disorders, baldness, Anti-cancer drug and other pharmacological activities. We report the presence of some of the significant components decided by GC-MS analysis, FT-IR analysis. From these results, it could be concluded that C. quadrangularis, C. halicacabum and D. viscosa contains different bioactive compounds. Assessment of pharmacological activity is under progress. Hence, it is prescribed as a plant of Phyto pharmaceutical significance.

9. Banu J, Varela E, Ali N, Soomro R, Kazi N, Fernandes G. Inhibition of Bone Loss by Cissus quadrangularisin Mice: A Preliminary Report. Journal of Osteoporosis, 2012, 10

10. Kumar R, Murugananthana G, Nandakumar K, Sahil Talwar. Isolation of anxiolytic principle from ethanolic root extract of Cardiospermum halicacabum. Phytomedicine. 2011; 18(23):219-223

11. Sheeba MS, Asha VV. Cardiospermum halicacabum ethanol extract inhibits LPS induced COX-2, TNF- and iNOS expression, which is mediated by NF-B regulation, in RAW264.7 cells. Journal of Ethnopharmacology, 2009; 124 (1):39-44.

12. Waako BPJ, Gumede P,. Smith PI. The in vitro and in vivo anti-malarial activity of Cardiospermum halicacabum L. and Momordica foetida Schumch. Et Thonn. Journal of Ethnopharmacology, 2005; 99(1):37-143.

13. Huang MH, Huang SS,. Wang BS. Antioxidant and antiinflammatory properties of Cardiospermum halicacabum and its reference compounds ex vivo and in vivo, Journal of Ethnopharmacology, 2011; 133(2):743-750.

14. J. Sadique T. Chandra V, Thenmozhi, Elango V. 1987) Biochemical modes of action of Cassia occidentalis and Cardiospermum halicacabum in inflammation. Journal of Ethnopharmacology, 1987; 19(2):201-212.

15. Jeyadevi R, Sivasudha T, Rameshkumar A, James Harnly M, Long Ze L. Phenolic profiling by UPLC-MS/MS and hepato protective activity of Cardiospermum halicacabum against $\mathrm{CCl} 4$ induced liver injury in Wistar rats. Journal of Functional Foods, 2013; 5(1):289-298

16. Albaker SM, Saeed Al gash FA, Hamid Hanash S, Ali A, Hazmi A. Prevalence and Evaluation of Antimicrobial Activity of Dodonaea viscosa Extract and Antibacterial Agents against salmonella Spp. Isolated from Poultry. International Journal of Medical and Health Sciences, 2015; 2(1):901-908. 
17. Rashed K, Luo M, Zhang L, Zheng Y. Dodonaea viscosa (L.) extracts as anti-human immunodeficiency virus type-1 (HIV1) agents and phyto constituents Peak, Journal of Medicinal Plant Research, 2013; 1(3):19-25.

18. Venkatesh S, Reddy YSR, Ramesh M, Swamy MM, Mahadevan N, Suresh B. Pharmacognostical studies on Dodonaea viscosa Leaves. African Journal of Pharmacy and Pharmacology, 2008; 2(4):83-88.
19. Teffo LS, Aderogba, Eloff JN. Antibacterial and antioxidant activities of four kaempferol methyl ethers isolated from Dodonaea viscosa Jacq. var. angustifolia leaf extracts. South African Journal of Botany, 2010; 76(1):25-29.

20. Duke JA. Database of Biologically Active Phytochemicals \& Their Activity, 1992, ISBN 9780849336713. 